\title{
Energy levels of semiconductor surface vacancies
}

\author{
M. S. Daw and D. L. Smith \\ California Institute of Technology, Pasadena, California 91125 \\ (Received 7 March 1980; accepted 15 April 1980)
}

\begin{abstract}
We present calculations of the bound state energy levels of anion vacancies near the surface of a III-V semiconductor. We consider the (110) surface of GaAs, InP, and the $\mathrm{Ga}_{1-x} \mathrm{Al}_{x}$ As alloy system. As the vacancy is moved toward the surface, the energy levels are only slightly perturbed until the vacancy reaches the second atomic layer from the surface. At this point, the anion vacancy levels move to lower energy. We find that there is a general trend in the vacancy energy levels with semiconductor ionicity. As the material becomes more ionic, the anion vacancy levels move to higher energy. Comparing this trend with experimentally observed Schottky barrier heights, we find a strong correlation between the position of the highest occupied level in the anion vacancy and the measured Fermi level at the surface. This result suggests that the recently proposed defect model is capable of accounting for observed trends in Schottky barrier formation.
\end{abstract}

PACS numbers: 73.20.Hb, 73.30. $+\mathrm{y}, 71.55 .-\mathrm{i}$

\section{INTRODUCTION}

It has recently been proposed that defects near the semiconductor surface determine the Schottky barrier height in III $\mathrm{V}$ semiconductors. ${ }^{1-4}$ In this model, these defects induce localized electronic states whose energies lie in the semiconductor band gap. These states then pin the Fermi level at the energy of the defect level. Motivated by this proposal, we investigate the energy levels of a simple vacancy near the surface of a III- $V$ semiconductor. Because anion material is found to be preferentially lost at the surface in several III-V semiconductors, we concentrate on anion vacancies. ${ }^{1-3,5,6}$ We compute the vacancy energy level as a function of distance from the (110) surface. We consider the position of the vacancy levels in III. V semiconductors of varying ionicity.

The paper is organized in the following way: in Sec. II, we discuss qualitatively the bound state energy levels of an anion vacancy in a III $-V$ semiconductor and the influence such vacancies would have on the surface Fermi level; in Sec. III we present our numerical results; our conclusions are contained in Sec. IV.

\section{ANION VACANCIES IN III-V SEMICONDUCTORS}

In the bulk of a III-V semiconductor, an ideal anion vacancy (no lattice relaxation) induces twofold and sixfold degenerate levels (including spin) with symmetry $a_{1}$ and $t_{2}$, respectively. The $a_{1}$ state occurs at lower energy than the $t_{2}$ state. There are three electrons associated with these bound (or resonant) levels in the neutral anion vacancy. Because the $t_{2}$ level is partially occupied (one electron) in the neutral vacancy, a Jahn-Teller distortion will occur. In Si, the vacancy with three electrons associated with the $a_{1}$ and $t_{2}$ states (positively charged in $\mathrm{Si}$ ) is known to undergo a tetragonal Jahn-Teller distortion. ${ }^{8}$ We assume that the distortion for the anion vacancy in the III. $V$ semiconductors is also tetragonal. In this case, the symmetry of the electronic states is reduced from $T_{d}$ to $D_{2 d}$ and the $t_{2}$ level splits into a twofold degenerate $b_{2}$ level and a fourfold degenerate $e$ level. The distortion is such that the $b_{2}$ level is at lower energy.

We consider three charge states of the anion vacancy $V^{+}$, $V^{0}$ and $V^{-}$with two, three and four electrons associated with the bound (or resonant) states, respectively. We describe the energies of these states in analogy with the vacancy in $S i, 8,9$ The energy zero is taken to be the $V^{+}$state with no tetragonal distortion ${ }^{10}$ and with the electrons which will bind to form the other charge states at the Fermi energy. The energies of the three states of interest can then be written as

$$
\begin{aligned}
E_{+}=\frac{1}{2} k Q^{2}, \quad E_{0} & =\frac{1}{2} k Q^{2}+\epsilon-V Q-\mu, \\
E_{-} & =\frac{1}{2} k Q^{2}+2(\epsilon-\mu-V Q)+u
\end{aligned}
$$

Here $Q$ is the displacement of a single atom in the tetragonal distortion, $k$ is the lattice restoring force constant for the displacement, $\epsilon$ is the one-electron energy (relative to the valence band maximum) of the $t_{2}$ state of the neutral vacancy with no lattice distortion, $V$ is the Jahn-Teller coupling coefficient for the distortion, ${ }^{10} u$ is the electrostatic repulsion for the negatively charged vacancy and $\mu$ is the electron chemical potential. Physically, $Q$ is fixed for each state to minimize the energy for that state. The minimum values for the energy are:

$$
\begin{array}{ll}
E_{+}=0, & E_{0}=\epsilon-\mu-V^{2} / 2 k \\
& E_{-}=2(\epsilon-\mu)-2 V^{2} / k+u
\end{array}
$$

For fixed $\mu$, the charge state of the vacancy is determined by the least of $E_{+}, E_{0}, E_{-}$. As pointed out in Ref. 9 , there is no value of $\mu$ for which the $V^{0}$ state is stable if $u<V^{2} / k$. For this condition, $V^{+}$is stable if $\mu<\epsilon-V^{2} / k+u / 2$ and the $V^{-}$is stable otherwise. If $u>V^{2} / k$, the neutral state may be stable. Under this condition, the $V^{+}$is stable if $\mu<\epsilon-V^{2} / 2 k, V^{0}$ if $\epsilon-V^{2} / 2 k<\mu<\epsilon+u-3 V^{2} / 2 k$, and $V-$ if $\mu>\epsilon+u-$ $3 V^{2} / 2 k$.

In the bulk, the chemical potential is fixed by shallow dopants. However, in the defect model for Schottky barrier 
formation, it is assumed that there is a large concentration of defects in a thin layer near the semiconductor surface. In this case, the position of the Fermi level relative to the band edges at the surface will be determined by the defects. For the case of a free surface (without a macroscopic metal overlayer) the defects near the surface will charge so as to compensate the charge in the dopletion region of the semiconductor. From electrostatics, the Fermi level measured relative to the valence band maximum at the surface, $\mu_{s}$, in $n$-type material is given by

$$
\mu_{s}=\mu-\frac{2 \pi e^{2}}{\epsilon_{0}} \frac{\left(N_{+}-N_{-}\right)^{2}}{n_{d}}
$$

where

$$
\left(N_{+}-N_{-}\right)=N_{v}\left[\frac{e^{-E_{+} / k T}-e^{-E_{-} / k T}}{e^{-E_{+} / k T}+e^{-E_{0} / k T}+e^{-E_{-} / k T}}\right] .
$$

Here $n_{d}$ is the donor concentration, $\epsilon_{0}$ is the semiconductor dielectric constant and $N_{+}, N_{-}$and $N_{v}$ are the number per unit area of positively charged, negatively charged and all vacancies, respectively. The vacancy energies are given in Eq. (2) where the one electron energy $c$ is measured relative to the surface band edges. In $p$-type material, $n_{d}$ in Eq. (3a) is replaced with $\left(-n_{a}\right)$.

For a Schottky barrier with a macroscopic metal overlayer, the defects charge and form a dipole with their image charge in the metal. The charge state of the defects need not be opposite to that in the space charge region because the metal overlayer can screen the space charge. Taking the vacancies to lie in a plane a distance $/$ from the metal surface, $\mu_{*}$ in $n$ type material is given by

$\mu_{s}=\left(I_{s}-\chi_{m}\right)+D_{I}+\frac{4 \pi e^{2} l}{\epsilon_{d}}\left[\left(N_{+}-N_{-}\right)+n_{d} x_{d}\right]$

Here $I_{s}$ is the semiconductor ionization potential $\chi_{m}$ is the metal work function, $x_{d}$ is the depletion length, $D_{I}$ is a chemically induced dipole layer which may occur at the interface ${ }^{11}$ and $\epsilon_{d}$ is the dielectric constant of the disordered region at the interface. In $p$-type material $n_{d l} \mathrm{in} \mathrm{Eq}$. (4) is replaced with $\left(-n_{a}\right)$.
At room temperature, $k T$ is very small compared to the cnergy scale of interest. Thus, the function $\left(N_{+}-N_{-}\right)$will charge very rapidly as $\mu_{s}$ moves past the stability points for the vacancy charge states. As a result, for a sufficiently high surface density of vacancies, $\mu_{s}$ will be pinned at onc of the stability points. In Table I, we list the stability points at which the pinning occurs and the minimum surface density of vacancies required to pin for the various possibilities. ${ }^{12}$ From this table, we see that the vacancy charge state and hence the relevant stability point at the free surface depends on the semiconductor doping. However, in the Schotky barrier case, the vacancy charge state and relevant stability point depends on $\left(I_{s}-\chi_{m}+D_{l}\right)$. Consequently, it is possible that $\mu_{s}$ may differ for the free surface of an $n$ - and $p$-lype semiconductor, 3,4 but doping does not significantly influence $\mu_{s}$ in a macroscopic Schot tky barrier.

In order that $\mu_{s}$ be approximately equal for metals in which $\left(I_{s}-\chi_{m}+D_{I}\right)$ has opposite signs, it is necessary to charge the defect region with opposite polarities. Simple donors and acceptors can charge with only one sign. Thus a single donor or acceptor defect cannot account for the independence of $\mu_{s}$ on $\chi_{m}$ observed in the $11-V$ semiconductors, whereas a level which can charge with either sign (such as the anion vacancy) can accoun for this observation.

\section{UE. RESUETS FOR ANION VACANCY LEVELS}

In order to determine $\mu_{s}$, it is necessary to know the parameters $\epsilon, V, k$ and $u$. In this section we estimate these parameters for various III-V semiconductors. We consider materials of different ionicity ${ }^{13}$ and attempt to correlate the results of our calculation with measured values for $\mu_{s}$. We find that $\epsilon$, for anion vacancies, increases with semiconductor ionicity in a manner similar to the experimentally observed behavior of $\mu_{s}, 3,4$ The other parameters are less strongly dependent on ionicity and lead to modest shifts in the position of the stability points. For example, in the calculations of Ref. 9 , the $V^{0}$ state in Si is found to be unstable and the shift in stability point $\left(-V^{2} / k+u\right)$ is $0.21 \mathrm{eV}$. Wo also determine the one electron energies for anion vacancies near the (110)

\begin{tabular}{|c|c|c|c|c|}
\hline & \multicolumn{2}{|c|}{$V^{0}$ stable } & \multicolumn{2}{|c|}{ No unstable } \\
\hline & Schottky barrier & Free surface & Schottky barrier & lree surface \\
\hline \multirow{4}{*}{$V^{-}$} & $\mu_{s}=\epsilon-\left(3 V^{2} / 2 k\right)+u$ & $\mu_{s}=\epsilon-\left(3 V^{2} / 2 k\right)+u$ & $\mu_{s}=\epsilon-\left(V^{2} / k\right)+(u / 2)$ & $\mu_{s}=\epsilon-\left(V^{2} / k\right)+(u / 2)$ \\
\hline & $N_{s}>\mu_{s}-\left(I_{s}-\chi_{m}+D_{H}\right)$ & $N>\left[n_{d} \epsilon_{0}\left(\mu-\mu_{s}\right)\right]^{1 / 2}$ & $\mu_{1}>\mu_{s}-I_{s}+x_{m}-D_{I}$ & $V_{s}>\left[n_{d} \epsilon_{0}\left(\mu-\mu_{s}\right)\right]^{1 / 2}$ \\
\hline & $N_{V}=\overline{4 \pi e^{2} l / \epsilon_{d}}$ & $N V=\sqrt[2 \pi e^{2}]{\mid}$ & $4 \pi e^{2} / / \epsilon_{d}$ & $N_{V}=2 \pi e^{2}$ \\
\hline & $\mu_{s} \geq I_{s}-\chi_{m}+D_{I}$ & $n$-type & $\mu_{s}>I_{s}-\chi_{m}+D_{l}$ & $n$-type \\
\hline \multirow[t]{2}{*}{$V^{0}$} & $\begin{array}{l}\mu_{s}=I_{s}-\chi_{m}+D_{l} \\
N_{V} \geq 0\end{array}$ & $\begin{array}{l}\mu_{s}=\mu^{2} \\
N_{V} \geq 0\end{array}$ & & \\
\hline & $\varepsilon-\frac{V^{2}}{2 k} \leq \mu_{s} \leq \epsilon-\frac{3 V^{2}}{2 k}+u$ & $\epsilon-\frac{V^{2}}{2 k} \leq \mu_{s} \leq \epsilon-\frac{3 V^{2}}{2 k}+u$ & & \\
\hline \multirow{4}{*}{$b^{+}$} & $\mu_{s}=\epsilon-\left(V^{2} / 2 k\right)$ & $\mu_{s}=\epsilon-\left(V^{2} / 2 k\right)$ & $\mu_{s}=\varepsilon-\left(V^{2} / k\right)+(u / 2)$ & $\mu_{s}=c-\left(V^{2} / k\right)+(u / 2)$ \\
\hline & $N_{V} \geq I_{s}-x_{m}+D_{l}-\mu_{s}$ & $N_{Y} \geq\left[n_{a} \epsilon_{0}\left(\mu_{s}-\mu\right)\right]^{1 / 2}$ & $N_{V} \geq l_{s}-\chi_{m}+D_{l}-\mu_{s}$ & $\left.N_{V} \geq n_{d} \epsilon_{0}\left(\mu_{s}-\mu\right)\right]^{1 / 2}$ \\
\hline & $4 \pi e^{2} l / \epsilon_{d}$ & $N y=\sqrt{2 \pi e^{2}}$ & $M=4 \pi e^{2 / \epsilon_{d}}$ & $M=\left[2 \pi e^{2}\right]$ \\
\hline & $\mu_{s} \leq I_{s}-X_{m}+D_{I}$ & $p$-type & $\mu_{s}<I_{s}-\chi_{m}+D_{I}$ & $p$-type \\
\hline
\end{tabular}

TABLEI. Surface Fermi level and surface vacancy concentration to complete pinning for various conditions. 
semiconductor surface. In this case, the highest occupied level in the neutral vacancy is the state of interest. It is partially filled (one electron in a twofold degenerate state) and is the state which will be involved in charging the vacancy.

Our calculations are performed in the tight binding approximation. We consider GaAs, InP, and $\mathrm{Ga}_{1-x} \mathrm{Al}_{x} \mathrm{As}$. Calculational details are included in Ref. 14. Our results for GaAs and InP are shown in Fig. 1. On the fifth layer from the surface, the energy levels are essentially the same as those in the bulk of the semiconductor. There are two bound (or resonant) levels; the $a_{1}$ level is occupied with two electrons and the higher $t_{2}$ level has one electron in the neutral vacancy. As the vacancy is moved toward the surface, there is no significant change ${ }^{15}$ in the energy levels until the vacancy reaches the second atomic layer from the surface. There the $t_{2}$ level splits into three nondegenerate levels and they move to lower energy. The arrow indicates the highest occupied level in the neutral vacancy. For the vacancy on the surface layer, one of the bound state levels is lost. The loss occurs because only three bonds are broken in the surface vacancy and hence only three bound states can be made. The energy levels are first significantly perturbed at the second atomic layer because there a vacancy has a nearest neighbor on the surface layer. Because of reconstruction, the hybridization of the surface cation orbitals involved in the bonding is $s p^{2}$ rather than $s p^{3}$ as in the bulk. As a result, in forming the anion vacancy on the second atomic layer from the surface, a $s p^{2}$ bond is broken. The dangling $s p^{2}$ orbital is of lower energy than the dangling $s p^{3}$ orbitals making up the bulk vacancy states because the $s p^{2}$ orbital has a larger $s$ state component. On the surface layer,

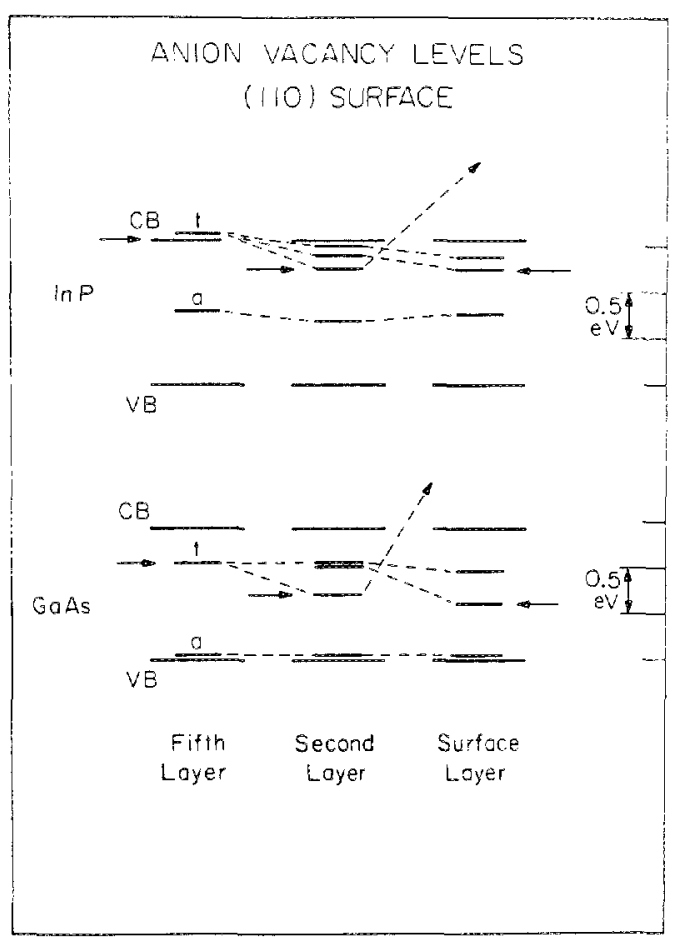

Fic. 1. Erergy positions, relative to the band edges, of ideal anion vacancies near the (110) surface of InP and GaAs. The bound state energy levels are essentially the same as those in the bulk until the vacancy reaches the second atomic layer from the surface. The arrows indicate the highest energy state occupied in the neutral vacancy.

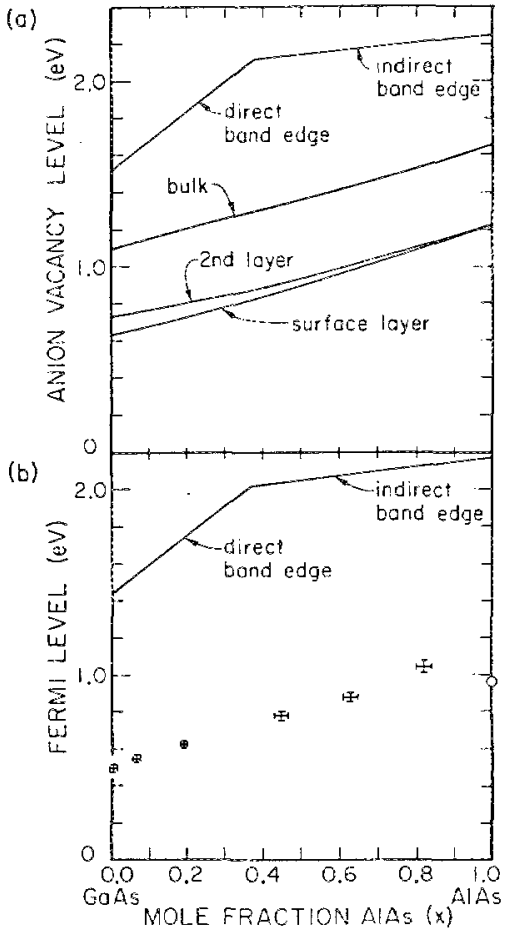

FIG. 2. (a) Position of the highest occupied state in the neutral anion vacancy as a function of alloy composition in $\mathrm{Ca}_{1-x} \mathrm{Al}_{x}$ As. Results for vacancies in the bulk, second atomic layer, and on the (110) surface are shown. The energy zero is the valence band maximum; the calculated position of the conduction band minimum is also shown

(b) Measure position of the surface Fermi level as a function of alloy composition in $\mathrm{Ga}_{1-x} \mathrm{Al} \mathrm{l}_{x} \mathrm{As}$. The measurements are from Ref. 16

two $s p^{2}$ bonds are broken and again the bound states are at lower energy than the bulk.

Comparing the results for $\mathrm{InP}$ with those of $\mathrm{GaAs}$, we see that the anion vacancy levels in InP are at higher energy relative to the band edges. This upward shift in the energy levels occurs because InP is more ionic than GaAs. In covalent materials, the gap between valence and conduction band states is primarily due to the splitting of bonding and antibonding states. In the more ionic materials, the valence band states are primarily anion-derived and the conduction band states are more strongly cation-derived. For the covalent case, the cation dangling bonds from the anion vacancy will appear between the bonding and antibonding states, that is, near midgap. For more ionic materials, the cation dangling bond states will move toward the cation-derived states of the conduction band. Thus the vacancy levels in GaAs occur at lower energy than the corresponding states in the more ionic InP. It is interesting to note that measured values of $\mu_{s}$ in III-V semiconductors show a trend similar to that calculated for the anion vacancy levels. In the series GaSb, GaAs, and InP (listed in order of increasing ionicity), $\mu_{s}$ is observed to increase from near the valence band maximum to near the conduction band minimum ${ }^{3,4}$

In Fig. 2, we show the calculated position of the highest occupied level in the neutral anion vacancy in the bulk, second atomic layer and on the $(110)$ surface of $\mathrm{Ga}_{1-x} \mathrm{Al} l_{x} \mathrm{As}$ as a function of alloy composition. The position of these levels, relative to the valence band maximum is an increasing function of the concentration of the more ionic AlAs. The energy of the highest occupied level in the neutral anion vacancy on the surface and second layers are quite close to each other, whereas this level on the third layer (not shown explicitly) is close to that of the bulk. This occurs because the vacancy states on the surface and second layers are made heavily from dangling bond orbitals of a surface Ga which is 
$\mathrm{SP}^{2}$-hybridized whereas those in the third and deeper layers are made heavily from dangling bond orbitals on Ga atoms off the surface which are SP $^{3}$ hybrids. In Fig. 2 we also show the measurements of Ref. 16 for $\mu_{s}$ in $\mathrm{Ga}_{1-x} \mathrm{~A} \mathrm{~L}_{x} \mathrm{As}$ as a function of $x$. We note that there is a strong similarity between the calculated positions of the highest filled nevtral anion level and the measured position of $\mu_{s}$.

We estimate the Jahn-Teller coupling coefficients $V$ and the Coulomb repulsion $u$ in the bulk using perturbation theory. The unperturbed wave-function can be written as

$$
\psi_{\beta}(r)=\sum_{\alpha j} C_{\alpha j}^{\beta}\left(\epsilon_{\beta}\right) A_{\alpha j}(r)
$$

where

$$
C_{\alpha j}^{\beta}\left(\epsilon_{\beta}\right)=\frac{G_{a j ; \beta 0}\left(\epsilon_{\beta}\right)}{\left.\mid-G_{\beta 0 ; \beta 0}^{\prime}\left(\epsilon_{\beta}\right)\right]^{1 / 2}} .
$$

Here $\alpha$ labels an atomic function and $j$ gives the atomic position, $G$ is the Green's function, $\epsilon_{\beta}$ is the energy of the unperturbed state (a $t_{2}$ state is of interest here, in this case $\beta$ indicates a $P$ orbital), the prime in Eq. (5b) indicates an energy derivative and $A$ is an atomic function. The Jahn-Teller coupling coefficient is calculated from

$$
V Q=\left\langle\psi_{\beta}\left|\frac{\partial H}{\partial Q} Q\right| \psi_{\beta}\right\rangle,
$$

where $Q$ is the tetragonal normal mode displacement. The distortion potential, $\partial H / \partial Q$ is found by assuming that the tight binding parameters scale like the overlap of the atomic functions. For GaAs and AlAs, the tetragonal distortion coupling coefficients for the $b_{2}$ state are $1.3 \mathrm{eV} / \vec{A}$ and $0.7 \mathrm{eV} / \vec{A}$, respectively. The $e$ states shift in the opposite direction by half those amounts. The Coulombic repulsion was estimated as

$$
u=\left\langle\psi_{\beta}\left(r_{1}\right) \psi_{\beta}\left(r_{2}\right)\left|\frac{e^{2}}{\epsilon\left(r_{12}\right) \mid r_{12}}\right| \psi_{\beta}\left(r_{1}\right) \psi_{\beta}\left(r_{2}\right)\right\rangle
$$

where $\left[e^{2} / \epsilon(r) r\right]$ is the Fourier transf orm of $\left(4 \pi e^{2} / \epsilon(q) q^{2}\right)$ and $\varepsilon(q)$ is the dielectric function. One center integrals on the first and second atomic shells were included in the estimate of $u$. The results were $0.20 \mathrm{eV}$ and $0.27 \mathrm{eV}$ for GaAs and AlAs, respectively. The force constant $k$, was estimated by sealing the Keating model calculations of hef. 9 for $\mathrm{Si}$ by the bond stretching force constant determined from compressibility. ${ }^{17}$ The result of this scaling was $12 \mathrm{eV} / \AA^{2}$ for both GaAs and AlAs (compared with $14.8 \mathrm{eV} / \AA^{2}$ for $\mathrm{Si}^{9}$ ).

These values for the parameters indicate that there is a small stability region for the neutral anion vacancy in GaAs and AlAs. However the accuracy of the estimates is not great enough to settle this issue. From our point of view, the important thing is that the qualitative behavior of the one electron energies shown in Fig. 2 is not greatly modified. For the $V^{+}$stability point the GaAs end point is shifted down by 0.07 $\mathrm{eV}$ and the AlAs end point down by $0.02 \mathrm{eV}$ as compared with Fig. 2 while for the $V^{-}$stability point the GaAs end point is unchanged and the AlAs endpoint is shifted up by $0.2 \mathrm{eV}$. Thus in both cases the slope of the bulk line in Fig. 2 is slightly increased. We are currently investigating the effects of lattice relaxation for vacancies on the surface. We expect results analogous to those in the buik.
Hence, with Jahn-Teller distortions and Coulomb corrections included, we still see a strong similarity between the calculated position of the anion vacancy levels and the measured values for $\mu_{\mathrm{s}}$. There is, of course, some uncertainty in the absolute energy levels but we think that the trends are significant.

\section{CONCLUSIONS}

We presented a calculation of energy levels of anion vacancies close to the (110) surface of $I I-V$ semiconductors. We have found that energy levels are not significantly changed until the vacancy reaches the second atomic layer from the surface. As one considers more ionic III-V semiconductors, the anion vacancy levels move to higher energy relative to the valence band maximum. There is a correlation between the calculated arion vacancy levels and the measured values of $\mu_{s}$ in III $-\mathrm{V}$ semiconductors. This correlation will probably be maintained if one considers other defect levels formed from cation dangling bonds. This correlation suggests that the defect model is a promising model of Schottky barrier formation.

\section{ACKNOWLEDGMENTS}

We thank T. C. McGill, W. E. Spicer, H. H. Wieder, and R. H. Williams for valuable discussions on subjects related to this work and for sending us preprints of their papers prior to publication. We acknowledge the support of the Office of Naval Research under Contract No. N00014-79-C-0797. D. L. Smith acknowledges support from the Alfred P. Sloan Foundation.

1. Lindau, P. W. Chye, C. M. Garner, P. Pianetta, C. Y. Su, and W. E. Spicer, 3. Vac. Sci. Technol. 15, 1332 (1978).

2P. W. Chye, I. Lindan, P. Pianetia, C. M. Gamer, C. Y. Su, and W. E. Spicer, Phys. Rev. B 18, 5545 (1978).

W. E. Spicer, P. W. Chye, P. R. Skeath, C. Y. Su, and I. Lindau, I. Vac. Sei Technol. 16, 1422 (1979).

${ }^{4}$ W. E. Spicer, I. Lindau, P. R. Skeath, C. Y. Su, and P. W. Chye, Phys. Rev Lett. $44,420(1980)$.

5V. Montgomery, R. H. Williams, and R. R. Varma, J. Phys. C 11, 1989 (1978).

'V. Montgomery, A. McKinley, and R. H. Williams (to be published).

7See, for example, J. Bernholc and S. T. Pantelides, Phys. Rev. B 18, 1780 (1978).

${ }^{8}$ G. D. Watkins, in Lattice Defects in Semiconductors-1974, edited by $F$. A. Huntley, Institute of Physics, Conference Series No. 23 (institute of Physies, Bristol and Iondon, 1975) p. 1.

'G. A. Baraff, E. O. Kane, and M. Schlüter, Phys. Rev. Lett. 43, 956 (1979).

${ }^{10}$ We neglect breathing mode disturtions.

1i. J. Brillson, Phys. Rev. B18, 2431 (1978).

${ }^{12}$ We neglect the term $n_{d} x_{d}$ in Fq. (4). This term is small.

${ }^{13}$ We use the ionicity parameter defined by E. J. Mele and I. D. Joannopoulous, Phys. Rev. 1817,1528 (1978). This parameter is based on atomic energies and can be incorporated into tight binding calculations in a natural way.

in. S. Daw and D. L. Smith, Phys. Rev. B 20, 5150 (1979).

${ }^{15} \mathrm{We}$ define $0.1 \mathrm{eV}$ as significant.

16J. S. Best, Appl. Phys. Lett. 34, 522 (1979).

17. Waser and I. Pauling. J. Chem. Phys. 18, 747 (1950). 